PROCEEDINGS OF THE

AMERICAN MATHEMATICAL SOCIETY

Volume 139, Number 10, October 2011, Pages 3691-3696

S 0002-9939(2011)11002-5

Article electronically published on March 30, 2011

\title{
ON CONVERGENCE RATES FOR SOLUTIONS OF APPROXIMATE MEAN CURVATURE EQUATIONS
}

\author{
HIROYOSHI MITAKE
}

(Communicated by Matthew J. Gursky)

\begin{abstract}
Evans and Spruck (1991) considered an approximate equation for the level-set equation of the mean curvature flow and proved the convergence of solutions. Deckelnick (2000) established a rate for the convergence. In this paper, we will provide a simple proof for the same result as that of Deckelnick. Moreover, we consider generalized mean curvature equations and introduce approximate equations for them and then establish a rate for the convergence.
\end{abstract}

\section{INTRODUCTION}

We are concerned with the initial value problem for the level-set equation of the mean curvature flow

$$
\begin{cases}u_{t}-\operatorname{tr}\left(b(D u) D^{2} u\right)=0 & \text { in } \mathbb{R}^{N} \times(0, \infty), \\ u(\cdot, 0)=u_{0} & \text { in } \mathbb{R}^{N},\end{cases}
$$

where $u$ is the real-valued unknown function on $\mathbb{R}^{N} \times[0, \infty), u_{t}:=\partial u / \partial t, D u:=$ $\left(\partial u / \partial x_{1}, \ldots, \partial u / \partial x_{n}\right), b(p):=I-(p \otimes p) /|p|^{2}$ for all $p \in \mathbb{R}^{N} \backslash\{0\}$, where $I$ is the unit matrix. It is worth mentioning that it is difficult to investigate (1), since $b(p)$ has a singularity at $p=0$ and it is degenerate for $p \neq 0$. Indeed, $b(p) p=0$ for any $p \in \mathbb{R}^{N} \backslash\{0\}$.

Evans and Spruck [7] and Chen, Giga and Goto [2, 3] established existence and uniqueness results in the context of the theory of viscosity solutions (see 4 for instance). In [3] the solution was constructed by Perron's method, while in [7, 2] the solution is constructed by approximating (1) by uniformly parabolic equations. In particular, the authors in [7] considered an approximate equation

$$
\begin{cases}u_{t}-\operatorname{tr}\left(b_{\varepsilon}(D u) D^{2} u\right)=0 & \text { in } \mathbb{R}^{N} \times(0, \infty), \\ u(\cdot, 0)=u_{0} & \text { in } \mathbb{R}^{N},\end{cases}
$$

for $\varepsilon>0$, where $b_{\varepsilon}(p)=I-(p \otimes p) /\left(|p|^{2}+\varepsilon^{2}\right)$ and then showed that solutions $u_{\varepsilon}$ of (2) converge locally uniformly on $\mathbb{R}^{N} \times[0, \infty)$ to the (unique) viscosity solution $u$ of (11) as $\varepsilon \rightarrow 0$. We note that (2) has neither singularities nor degeneracies for

Received by the editors August 25, 2010.

2010 Mathematics Subject Classification. Primary 53C44, 65M15, 35D40.

Key words and phrases. Mean curvature equation, affine curvature equation, approximate equations, convergence rate.

This work was partially supported by the Research Fellowship (22-1725) for Young Researcher from JSPS.

(C)2011 American Mathematical Society Reverts to public domain 28 years from publication 
any $\varepsilon>0$ and therefore it is easier to deal with (2) from the point of view of the theory of PDE.

From the point of view of numerical analysis, (2) is important too. Indeed, Crandall and Lions in 5 gave an explicit finite difference scheme which is both monotone and consistent by introducing a scheme for (2) and by using the convergence $u_{\varepsilon} \rightarrow u$ as $\varepsilon \rightarrow 0$ rather than discretizing (11) directly. Deckelnick in 6] derived an $L^{\infty}$-error estimate between the numerical solution and the viscosity solution of (1) by using the convergence rate of $u_{\varepsilon} \rightarrow u$. Deckelnick has already obtained essentially the same convergence rate result as that in Theorem 1, but we emphasize that the proof in [6] seems to be more technical and we give a simple proof in this paper. Moreover, we give the constant appearing in the convergence rate result more explicitly.

We make the following assumption on $u_{0}$ throughout this paper:

$$
u_{0} \in W^{1, \infty}\left(\mathbb{R}^{N}\right), \quad\left|u_{0}(x)\right| \leq 1 \text { for all } x \in \mathbb{R}^{N} \text { and }
$$

there exists $R_{0}>0$ such that $u_{0}(x)=-1$ for all $x \in \mathbb{R}^{N} \backslash B\left(0, R_{0}\right)$,

where $B\left(0, R_{0}\right):=\left\{x \in \mathbb{R}^{N}|| x \mid<R_{0}\right\}$. Then it is known that the solution of (11) is Lipschitz continuous with a Lipschitz constant $\left\|D u_{0}\right\|_{\infty}$ with respect to the $x$-variable and $u(x, t)=-1$ for all $(x, t) \in\left(\mathbb{R}^{N} \backslash B\left(0, R_{0}+\sqrt{2}\right)\right) \times[0, \infty)$, where $\|\cdot\|_{\infty}=\|\cdot\|_{L^{\infty}\left(\mathbb{R}^{N}\right)}($ see [7, 8] $)$.

In Section 2 we prove the convergence rate result for (1), and in Section 3 we consider its generalization.

\section{Convergence rate for approximate mean Curvature equation}

We prove in this section

Theorem 1. Let $u$ and $u_{\varepsilon}$ be the viscosity solutions of (11) and (2), respectively. Then there exists $C>0$ which depends only on $N$ such that

$$
\left\|\left(u-u_{\varepsilon}\right)(\cdot, t)\right\|_{\infty} \leq C\left\|D u_{0}\right\|_{\infty}^{\frac{k}{2(k-1)}} \sqrt{k t} \varepsilon^{\frac{k-2}{2(k-1)}}
$$

for $t>0, k \in \mathbb{N}$ with $k \geq 4$ and $\varepsilon>0$.

Proof. Fix $T>0$ and $k \in \mathbb{N}$ with $k \geq 4$. Let $\delta \in(0,1)$ and $K>0$, which will be fixed later. We consider

$$
\sup _{x, y \in \mathbb{R}^{N}, t \in[0, T]}\left\{u(x, t)-u_{\varepsilon}(y, t)-\frac{|x-y|^{k}}{\delta^{k}}-K t\right\},
$$

and then the supremum is attained at some $(\bar{x}, \bar{y}, \bar{t}) \in \bar{B}\left(0, R_{0}+\sqrt{2}\right)^{2} \times[0, T]$.

We consider the case where $\bar{t} \in(0, T]$. In view of Ishii's lemma (see [4] for instance), for any $\rho>0$ there exist $(a, p, X) \in \bar{J}^{2,+} u(\bar{x}, \bar{t})$ and $(b, p, Y) \in \bar{J}^{2,-} u_{\varepsilon}(\bar{y}, \bar{t})$ such that

$$
a-b=K, \quad p=\frac{k|\bar{x}-\bar{y}|^{k-2}}{\delta^{k}}(\bar{x}-\bar{y}),\left(\begin{array}{cc}
X & 0 \\
0 & -Y
\end{array}\right) \leq A+\rho A^{2},
$$


where $\bar{J}^{2,+} f(x, t)$ (resp., $\left.\bar{J}^{2,-} f(x, t)\right)$ is the closure of the superjet (resp., subjet) of a function $f$ at $(x, t)$ and

$$
\begin{aligned}
A:= & \frac{k}{\delta^{k}}|\bar{x}-\bar{y}|^{k-2}\left(\begin{array}{cc}
I & -I \\
-I & I
\end{array}\right) \\
& +\frac{k(k-2)}{\delta^{k}}|\bar{x}-\bar{y}|^{k-4}\left(\begin{array}{cc}
(\bar{x}-\bar{y}) \otimes(\bar{x}-\bar{y}) & -(\bar{x}-\bar{y}) \otimes(\bar{x}-\bar{y}) \\
-(\bar{x}-\bar{y}) \otimes(\bar{x}-\bar{y}) & (\bar{x}-\bar{y}) \otimes(\bar{x}-\bar{y})
\end{array}\right) .
\end{aligned}
$$

The definition of viscosity solutions immediately implies the following inequality:

$$
K-\operatorname{tr}(b(p) X)^{*}+\operatorname{tr}\left(b_{\varepsilon}(p) Y\right) \leq 0,
$$

where $*$ denotes the upper-semicontinuous envelope.

In the case where $p=0$, we have $\bar{x}=\bar{y}$. Therefore we have $A=0, X \leq 0$ and $Y \geq 0$, which implies that $\operatorname{tr}(b(p) X)^{*}-\operatorname{tr}\left(b_{\varepsilon}(p) Y\right) \leq 0$, which contradicts that $K>0$. We consider the case where $p \neq 0$. Note that $b^{1 / 2}(p)=b(p)$ and

$$
b_{\varepsilon}^{1 / 2}(p)=I-\frac{p \otimes p}{\langle p\rangle_{\varepsilon}\left(\langle p\rangle_{\varepsilon}+\varepsilon\right)}
$$

for all $p \in \mathbb{R}^{N}$, where $\langle p\rangle_{\varepsilon}:=\sqrt{|p|^{2}+\varepsilon^{2}}$. Setting $a:=\operatorname{tr}(b(p) X)-\operatorname{tr}\left(b_{\varepsilon}(p) Y\right)$, we calculate that

$$
\begin{aligned}
a & =\sum_{i=1}^{N}\left\{\left\langle X b^{1 / 2}(p) e_{i}, b^{1 / 2}(p) e_{i}\right\rangle-\left\langle Y b_{\varepsilon}^{1 / 2}(p) e_{i}, b_{\varepsilon}^{1 / 2}(p) e_{i}\right\rangle\right\} \\
& \leq \sum_{i=1}^{N}\left\langle A\left(\begin{array}{c}
b^{1 / 2}(p) e_{i} \\
b_{\varepsilon}^{1 / 2}(p) e_{i}
\end{array}\right),\left(\begin{array}{c}
b^{1 / 2}(p) e_{i} \\
b_{\varepsilon}^{1 / 2}(p) e_{i}
\end{array}\right)\right\rangle+\rho\left|A^{2}\right|,
\end{aligned}
$$

where $|\cdot|$ is a matrix norm and

$$
\begin{aligned}
\sum_{i=1}^{N}\left\langle A\left(\begin{array}{c}
b^{1 / 2}(p) e_{i} \\
b_{\varepsilon}^{1 / 2}(p) e_{i}
\end{array}\right),\left(\begin{array}{c}
b^{1 / 2}(p) e_{i} \\
b_{\varepsilon}^{1 / 2}(p) e_{i}
\end{array}\right)\right\rangle & \leq \frac{N k(k-1)}{\delta^{k}}|\bar{x}-\bar{y}|^{k-2}\left|b^{1 / 2}(p)-b_{\varepsilon}^{1 / 2}(p)\right|^{2} \\
& \leq \frac{N k(k-1)}{\delta^{k}}\left(\frac{\delta^{k}|p|}{k}\right)^{\frac{k-2}{k-1}} \frac{\varepsilon^{2}}{|p|^{2}+\varepsilon^{2}} \\
& \leq C_{1}(k-1) \delta^{\frac{-k}{k-1}} \frac{\varepsilon^{2}|p|^{\frac{k-2}{k-1}}}{|p|^{2}+\varepsilon^{2}},
\end{aligned}
$$

where $C_{1}$ is a constant which depends only on $N$. Set

$$
f_{\varepsilon}(r):=\frac{\varepsilon^{2} r^{\frac{k-2}{k-1}}}{r^{2}+\varepsilon^{2}} .
$$

Then we have $f_{\varepsilon}(r) \leq \varepsilon^{\frac{k-2}{k-1}}$ for all $r \geq 0$. Therefore sending $\rho \rightarrow 0$ in (6) yields

$$
a \leq C_{1}(k-1) \delta^{-\frac{k}{k-1}} \varepsilon^{\frac{k-2}{k-1}} .
$$

Putting $K=C_{1} k \delta^{-\frac{k}{k-1}} \varepsilon^{\frac{k-2}{k-1}}$, we necessarily have $\bar{t}=0$. Since we have

$$
|\bar{x}-\bar{y}| \leq\left\|D u_{0}\right\|_{\infty}^{\frac{1}{k-1}} \delta^{\frac{k}{k-1}}
$$


in view of the Lipschitz continuity of $u$, we have

$$
\begin{aligned}
\left(u-u_{\varepsilon}\right)(x, t) & \leq u(\bar{x}, 0)-u_{\varepsilon}(\bar{y}, 0)-\frac{|\bar{x}-\bar{y}|^{k}}{\delta^{k}}+K t \\
& \leq\left\|D u_{0}\right\|_{\infty}^{\frac{k}{k-1}} \delta^{\frac{k}{k-1}}+C_{1} k \delta^{-\frac{k}{k-1}} \varepsilon^{\frac{k-2}{k-1}} t
\end{aligned}
$$

for any $(x, t) \in \mathbb{R}^{N} \times[0, \infty)$. By optimizing with respect to $\delta$, we get the conclusion.

\section{Generalization}

We consider in this section the level-set equation of the evolution of compact hypersurfaces $\left\{\Gamma_{t}\right\}_{t \geq 0} \subset \mathbb{R}^{N}$ moving according to the law $V=\kappa^{\alpha}$ for $\alpha=1 /(2 n-1)$ with any $n \in \mathbb{N}$ or $V=\kappa_{+}^{\alpha}$ for any $\alpha \in(0,1]$, where $V$ is the normal velocity of $\Gamma_{t}$ and $\kappa$ is the mean curvature; i.e.,

$$
u_{t}=\operatorname{tr}\left(b(D u) D^{2} u\right)^{\alpha}|D u|^{1-\alpha} \text { in } \mathbb{R}^{N} \times(0, \infty)
$$

or

$$
u_{t}=\operatorname{tr}\left(b(D u) D^{2} u\right)_{+}^{\alpha}|D u|^{1-\alpha} \quad \text { in } \mathbb{R}^{N} \times(0, \infty) .
$$

Here $a(x)_{+}:=\max \{a(x), 0\}$ for any function $a$. Equations (9), (10) are one of the fundamental equations appearing in the image processing (see [1, 8, for instance). Note that in the case where $\alpha=1$, (9) corresponds to the mean curvature equation and in the case where $\alpha=1 / 3$, (9) or (10) is called the affine curvature equation. We note that these equations are included by the general geometric equations which are dealt with in [3, 9, 8, and therefore we know that there exists a unique viscosity solution of (9) or (10).

We introduce approximate equations for (9) and (10):

$$
u_{t}=\operatorname{tr}\left(b_{\varepsilon}^{\alpha}(D u) D^{2} u\right)^{\alpha}|D u|^{1-\alpha} \text { in } \mathbb{R}^{N} \times(0, \infty)
$$

and

$$
u_{t}=\operatorname{tr}\left(b_{\varepsilon}^{\alpha}(D u) D^{2} u\right)_{+}^{\alpha}|D u|^{1-\alpha} \quad \text { in } \mathbb{R}^{N} \times(0, \infty),
$$

where

$$
b_{\varepsilon}^{\alpha}(p)=P\left(\begin{array}{ccccc}
1 & 0 & 0 & \cdots & 0 \\
0 & \frac{\varepsilon^{2}}{|p|^{2 / \alpha}+\varepsilon^{2}} & 0 & \cdots & 0 \\
\vdots & \vdots & \vdots & \ddots & \vdots \\
0 & 0 & 0 & \cdots & \frac{\varepsilon^{2}}{|p|^{2 / \alpha}+\varepsilon^{2}}
\end{array}\right) P^{T}
$$

and $P$ is an orthogonal matrix which satisfies

$$
b(p)=P\left(\begin{array}{cccc}
1 & 0 & \cdots & 0 \\
0 & 0 & \cdots & 0 \\
\vdots & \vdots & & \vdots \\
0 & 0 & \cdots & 0
\end{array}\right) P^{T}
$$

We note that (11) and (12) have neither singularities nor degeneracies for any $\varepsilon>0$ and also that when $\alpha=1, b_{\varepsilon}^{1}(p)$ corresponds to $b_{\varepsilon}(p)$ in Sections 1, 2. Moreover we note that we can easily calculate the term $\left|\left(b^{\alpha}\right)^{1 / 2}(p)-\left(b_{\varepsilon}^{\alpha}\right)^{1 / 2}(p)\right|$, which will be helpful to get such an estimate (77). 
Finally we give a sketch of the proof of

Theorem 2. Let $\alpha>0$ and $u, u_{\varepsilon}$ be the viscosity solutions of the initial value problems of (91) and (11) or (10) and (12) with the initial value $u_{0}$, respectively. Then there exists $C>0$ which depends only on $N, \alpha$ such that

$$
\left\|\left(u-u_{\varepsilon}\right)(\cdot, t)\right\|_{\infty} \leq C\left\|D u_{0}\right\|_{\infty}^{\frac{k}{k-1} \cdot \frac{\alpha}{1+\alpha}} k^{\frac{\alpha}{1+\alpha}} t^{\frac{1}{1+\alpha}} \varepsilon^{\left(1-\frac{\alpha}{k-1}\right) \frac{\alpha}{1+\alpha}}
$$

for $t>0, k \in \mathbb{N}$ with $k \geq 4$ and $\varepsilon>0$.

Sketch of Proof. We only consider equations (9) and (11). Let $\delta, K, \bar{x}, \bar{y}, \bar{z}, p$ and $A$ be the same as those in the proof of Theorem 10 Noting that $r^{\alpha}-s^{\alpha} \leq 2(r-s)^{\alpha}$ for any $r, s \in \mathbb{R}$, we have

$$
K \leq C_{1}(k-1)^{\alpha} \delta^{\frac{-\alpha k}{k-1}} \frac{\varepsilon^{2 \alpha}|p|^{\frac{\alpha(k-2)}{k-1}}}{\left(|p|^{2}+\varepsilon^{2}\right)^{\alpha}}|p|^{1-\alpha}+\rho\left|A^{2}\right|
$$

for some constant $C_{1}$ which depends only on $\alpha, N$. Setting

$$
f_{\varepsilon}^{\alpha}(r):=\frac{\varepsilon^{2 \alpha} r^{1-\alpha+\frac{\alpha(k-2)}{k-1}}}{\left(r^{\frac{2}{\alpha}}+\varepsilon^{2}\right)^{\alpha}},
$$

we have $f_{\varepsilon}^{\alpha}(r) \leq C_{2} \varepsilon^{\alpha\left(1-\frac{\alpha}{k-1}\right)}$ for all $r \geq 0$ and some $C_{2}>0$ which depends only on $\alpha$. Sending $\rho \rightarrow 0$ yields

$$
K \leq C_{3}(k-1)^{\alpha} \delta^{-\frac{\alpha k}{k-1}} \varepsilon^{\alpha\left(1-\frac{\alpha}{k-1}\right)},
$$

where $C_{3}$ is a constant which depends only on $N, \alpha$.

Putting $K=C_{3} k^{\alpha} \delta^{-\frac{\alpha k}{k-1}} \varepsilon^{\alpha\left(1-\frac{\alpha}{k-1}\right)}$, we necessarily have $\bar{t}=0$. Therefore by (8) we have

$$
\begin{aligned}
\left(u-u_{\varepsilon}\right)(x, t) & \leq u(\bar{x}, 0)-u_{\varepsilon}(\bar{y}, 0)-\frac{|\bar{x}-\bar{y}|^{k}}{\delta^{k}}+K t \\
& \leq\left\|D u_{0}\right\|_{\infty}^{\frac{k}{k-1}} \delta^{\frac{k}{k-1}}+C_{3} k^{\alpha} \delta^{-\frac{\alpha k}{k-1}} \varepsilon^{\alpha\left(1-\frac{\alpha}{k-1}\right)} t
\end{aligned}
$$

for any $(x, t) \in \mathbb{R}^{N} \times[0, \infty)$. By optimizing with respect to $\delta$, we get the conclusion.

\section{ACKNowledgements}

The author is grateful to Professors Guy Barles and Espen R. Jakobsen for their fruitful discussions, as well as to Professor Yoshikazu Giga for useful comments on the existence results for (11), (11) or (12). This work was partially done while the author was visiting the Laboratoire de Mathématiques et Physique Théorique, Université de Tours. He is grateful for its hospitality.

\section{REFERENCES}

1. L. Alvarez, F. Guichard, P.-L. Lions, J.-M. Morel, Axioms and fundamental equations of image processing, Arch. Rational Mech. Anal. 123 (1993), no. 3, 199-257. MR1225209 (94j:68306)

2. Y. G. Chen, Y. Giga, S. Goto, Uniqueness and existence of viscosity solutions of generalized mean curvature flow equations, Proc. Japan. Acad. 65 (1989), 207-210. MR1030181 (91b:35049)

3. Y. G. Chen, Y. Giga, S. Goto, Uniqueness and existence of viscosity solutions of generalized mean curvature flow equations, J. Differential Geom. 33 (1991), no. 3, 749-786. MR.1100211 (93a:35093) 
4. M. G. Crandall, H. Ishii, P.-L. Lions, User's guide to viscosity solutions of second order partial differential equations, Bull. Amer. Math. Soc. (N.S.) 27 (1992), no. 1, 1-67. MR.1118699 $(92 \mathrm{j}: 35050)$

5. M. G. Crandall, P.-L. Lions, Convergent difference schemes for nonlinear parabolic equations and mean curvature motion, Numer. Math. 75 (1996), no. 1, 17-41. MR.1417861 (97j:65134)

6. K. Deckelnick, Error bounds for a difference scheme approximating viscosity solutions of mean curvature flow, Interfaces Free Bound. 2 (2000), no. 2, 117-142. MR.1760409 (2001g:65098)

7. L. C. Evans, J. Spruck, Motion of level sets by mean curvature. I, J. Differential Geom. 33 (1991), no. 3, 635-681. MR1100206 (92h:35097)

8. Y. Giga, Surface evolution equations. A level set approach, Monographs in Mathematics, 99. Birkhäuser Verlag, Basel, 2006. MR2238463 (2007j:53071)

9. Y. Giga, S. Goto, Motion of hypersurfaces and geometric equations, J. Math. Soc. Japan 44 (1992), 99-111. MR1139660 (93b:58025)

Department of Applied Mathematics, Graduate School of Engineering, Hiroshima UNIVERsity, Higashi-Hiroshima 739-8527, JAPAN

E-mail address: mitake@hiroshima-u.ac.jp 